\title{
Internal resonance vibration induced by nonlinearity of primary suspension system in high-speed vehicle system
}

\author{
Yu Biao Liu • Yun Lin Xing • S. S. Law • \\ Ying Ying Zhang
}

Received: 25 June 2016 / Accepted: 14 February 2017 / Published online: 17 March 2017

(C) Springer Science+Business Media Dordrecht 2017

\begin{abstract}
This paper studies the phenomenon of internal resonance in high-speed vehicle system under high frequency periodic excitations. A numerical model of the vehicle system, taking into consideration the dynamic effects of the primary suspension system and the flexibility of the car-body, is established for the study. An approximate approach incorporating the incremental harmonic balance method with frequency response function is adopted to solve the dynamic responses of the vehicle system in frequency domain. Numerical results show that internal resonance vibration in the vehicle system may occur with certain combinations of design parameters of the vehicle system.
\end{abstract}

\section{Y. B. Liu · Y. Y. Zhang}

Key Laboratory for mechanics in Fluid Solid Coupling Systems, Institute of Mechanics, Chinese Academy of Sciences, Beijing, People's Republic of China e-mail: yubiao@imech.ac.cn

Y. Y. Zhang

e-mail: zhangying6971@ 126.com

Y. L. Xing $(\bowtie)$

Beijing Engineering Technology Research Center for Micro-Vibration Environmental Control, China Electronics Engineering Design Institute, Beijing,

People's Republic of China

e-mail: xingyunlin@yeah.net

\section{S. S. Law}

Department of Civil and Environmental Engineering, The Hong Kong Polytechnic University, Hunghom, Kowloon, Hong Kong, People's Republic of China

e-mail: cesslaw@inet.polyu.edu.hk
The vibration of the car-body and the primary suspension system are significantly amplified with energy transmitted between the natural modes of the car-body and the primary suspension system. Parametric studies on the internal resonance are further explored. Results show that the nonlinearity of the primary suspension spring and the modal damping ratio of the vehicle system play very important roles to the occurrence of internal resonance.

Keywords High-speed vehicle · Incremental harmonic balance method - Nonlinear steady-state solution $\cdot$ Internal resonance

\section{Introduction}

The ultimate goals of modern vehicle engineering are to improve the riding comfort, to minimize the wear of the vehicle components, and most importantly, to ensure a safe operation. The vibration of a vehicle system is closely related to the above performance and safety issues and it has drawn considerable attention in the research and development of railway vehicle. On-line measurements of CRH trains in China [21,22] showed that there exist three discrete wavelengths in the wheel-track irregularity spectrum corresponding to the track slab length $(6.5 \mathrm{~m})$, half of the track slab length $(3.25 \mathrm{~m})$ and the circumference of the wheelset $(2.7 \mathrm{~m})$. The wheel-track excitation frequency corresponding to these wavelengths increases with the run- 
ning speed, and they are, respectively, 17.1, 34.2 and $41.1 \mathrm{~Hz}$ at a running speed of $400 \mathrm{~km} / \mathrm{h}$. With the operation of modern lightweight vehicle, the fundamental natural frequency of the primary suspension spring and the higher-order natural frequencies of the vehicle system would be lower and close to these excitation frequencies. One of these excitation frequencies would be close to the fundamental natural frequency of the primary suspension with variation of the running speed of vehicle. The vibration of the wheelsets may be amplified and transmitted to the bogies and car-body through the primary suspension. If these happen, the high frequency dynamic responses of the vehicle system could then be enlarged significantly $[6,15]$. On the other hand, the classical models of railway vehicle dynamics usually treat the primary suspension as a static spring without consideration of its dynamic effect. According to Lee's $[1,10]$ and Liu's $[11,12]$ research on the primary suspension springs, when the excitation frequencies are higher than $40 \mathrm{~Hz}$, the dynamic effect of the primary suspension should not be ignored. So the static assumption to the primary suspension spring is feasible with vehicles running at low speed, but is not appropriate when vehicles are running at high speed.

The steady-state response of a high-speed vehicle system with nonlinear primary suspension is studied in frequency domain in this paper, and the incremental harmonic balance method (IHB) is adopted for the frequency response analysis. The IHB method, developed by Lau and Cheung [8], is used to obtain the steadystate solution of the nonlinear system under harmonic excitations. Pierre and Dowell [14] generalized this method to analyze the harmonic responses of dry friction damped systems. Their results showed that the IHB method can yield very accurate steady-state results with some advantages over the time domain methods. Cheung et al. [5] derived the formulation of IHB method for a general system of differential equations with cubic nonlinearity. An incremental arc-length method combined with a cubic extrapolation technique was adopted to trace the response curve automatically. Sze et al. [19] applied the IHB method to study the nonlinear vibration of axially moving beams. Chen et al. [4] studied the nonlinear vibration of plane structures by introducing finite element in the IHB method. Lu and Lin [13] modified IHB method so that periodic motions of rotating disk can be determined as well as oscillatory periodic motions in a unified formulation. Pun et al. [16,17] analyzed the free and forced vibration behavior of an
L-shaped beam with a limit stop. Pun combined the IHB method with the approximate receptance of the flexible beam to examine the complex internal resonance vibration. Pun's analysis revealed the presence of multiple internal resonance vibration involving interactions among the first five modes and indicated that this mode interaction may occur if the low- and highorder harmonics were close to two modal frequencies at the same time. He also pointed out that the damping could effectively attenuate the internal resonance vibration. All studies mentioned above showed that the IHB method had certain advantages in obtaining the steady-state response of nonlinear system compared to the time domain methods.

The model of the high-speed vehicle taking consideration of the flexibility of the car-body and the dynamic effect of the primary suspension is established for the study. The influence of dynamic response of the primary suspension on the vehicle system and the internal resonance phenomenon are studied, and the interaction between the mode of the primary suspension and the flexural mode of the vehicle system is the main focuses of this paper. The conditions under which the internal resonance in the vehicle system may occur are also explored.

\section{Vehicle system modeling}

\subsection{Modeling of the car-body}

The car-body is modeled as a box-like girder supported on the secondary suspensions discretized into finite elements. Only the flexible bending modes are considered in the modeling. The modeling approach is similar to the method proposed by Zhou et al. [24]. The equations of motion of the system can be written in generally form as

$$
\begin{aligned}
\mathbf{M}_{c} \ddot{\mathbf{x}}+\mathbf{C}_{c} \dot{\mathbf{x}}+\mathbf{K}_{c} \mathbf{x} \\
\quad=\left\{0, \ldots, P_{1}, \ldots, 0, \ldots, P_{2}, \ldots, 0\right\}^{T}
\end{aligned}
$$

where $\ddot{\mathbf{x}}, \dot{\mathbf{x}}$ and $\mathbf{x}$ are the acceleration, velocity and displacement vectors and $\mathbf{M}_{c}, \mathbf{C}_{c}$ and $\mathbf{K}_{c}$ represent the mass, damping and stiffness matrices, respectively. $P_{1}$ is the force from the secondary suspension on the front bogie and $P_{2}$ is the force on the rear bogie. 
Fig. 1 The dynamic model of the primary suspension

\subsection{Primary suspension spring modeling}

The primary suspension spring is modeled as dispersed rigid blocks connected with a series of springs as shown in Fig. 1 [11,12]. Let $m_{s}$ and $K_{p z}$ denote the total mass and the linear stiffness and $N$ denote the number of dispersed rigid blocks which is taken as 50 in this study. The mass of the dispersed rigid blocks and stiffness of the dispersed springs attached are $m_{s n}=m_{s} / N$ and $K_{p z n}=(N+1) K_{p z}$.

It is noted that the primary suspension spring stiffness usually exhibits hardening characteristic [7,23]. In this study, two nonlinear spring elements with a cubic nonlinear stiffness are used to simulate this property. Let $K_{p 3}$ denote the coefficient of the cubic nonlinear hardening component. The constitutive relationship between the force $F$ and displacement $x$ of the suspension spring can be expressed as

$F=K_{p z} x+\frac{1}{8} K_{p 3} x^{3}$.

A dimensionless parameter is defined as

$\beta=\frac{1}{8} \frac{K_{p 3} x_{0}^{2}}{K_{p z}}$.

where the symbol $x_{0}$ represents the static displacement of the primary suspension spring in the static equi- librium position. Parameter $\beta$ represents the extent of nonlinear hardening. Since damping within the spring system is small, no damping is assumed. The dimensionless constitutive relationship can be expressed as

$f\left(\frac{x}{x_{0}}\right)=\left(\frac{x}{x_{0}}\right)+\beta\left(\frac{x}{x_{0}}\right)^{3}$

It is noted from Eq. 4 that the internal forces of the primary suspension springs have both linear and nonlinear stiffness components. Putting the nonlinear part on the right-hand-side, the equations of motion for the dynamic model of the primary suspension springs can be written as:

$$
\begin{aligned}
& \mathbf{M}_{s} \ddot{\mathbf{x}}_{s}+\mathbf{K}_{s} \mathbf{x}_{s}=-\mathbf{f}_{n s w}-\mathbf{f}_{n s b} \\
& \mathbf{f}_{n s w}=\left[f_{s w}, 0, \ldots,-f_{s w}, 0,0, \ldots, 0\right]^{T} \\
& \mathbf{f}_{n s b}=\left[0,0, \ldots, 0, f_{s b}, 0, \ldots,-f_{s b}\right]^{T}
\end{aligned}
$$

$$
\begin{aligned}
\mathbf{M}_{s}= & {\left[\begin{array}{lllll}
0 & & & \\
& m_{s n} & & \\
& \ddots & & \\
& & m_{s n} & \\
& & &
\end{array}\right] } \\
\mathbf{K}_{s}= & {\left[\begin{array}{cccccc}
K_{p z n} & -K_{p z n} & & & & \\
-K_{p z n} & 2 K_{p z n} & -K_{p z n} & & \\
& -K_{p z n} & 2 K_{p z n} & \ddots & & \\
& & \ddots & \ddots & \ddots & \\
& & & \ddots & 2 K_{p z n} & -K_{p z n} \\
& & & & -K_{p z n} & K_{p z n}
\end{array}\right] }
\end{aligned}
$$

where $f_{s w}=K_{p 3}\left(z_{w}-x_{s \frac{n}{2}}\right)^{3}$ and $f_{s b}=K_{p 3}$ $\left(x_{s \frac{n}{2}}-z_{b}\right)^{3}$ represent the nonlinear parts of the internal forces of the primary suspension springs, and $z_{w}, z_{b}$ and $x_{s \frac{n}{2}}$ denote the vertical displacements of the wheelset, the bogie and the mid-nodes of the primary suspension spring respectively. It can be seen from Eqs. 6 and 7 that the form of vectors $\mathbf{f}_{n s w}$ and $\mathbf{f}_{n s b}$ are identical except the internal force terms $f_{s w}$ and $f_{s b}$ which are in different position. The dispersed dampers in the vehicle system can also be treated in the same way by putting the internal forces vectors on the right-hand-side. The nonlinear spring elements and the dispersed dampers are referred to as the dispersed elements, and $\mathbf{f}_{l}\left(l=1,2, \ldots N_{e}\right)$ represents all the internal force vectors of the dispersed elements, and $N_{e}$ denotes the total number of the dispersed elements. 


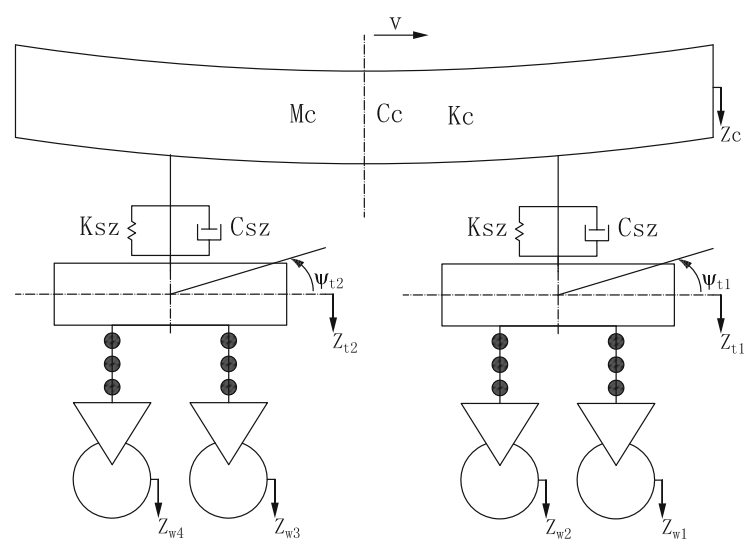

Fig. 2 Side view of the vehicle model

Suppose the $l$ th dispersed element connects to two degrees-of-freedoms (DOFs), those are the $l_{i}$ th DOF and the $l_{j}$ th DOF, then $\mathbf{f}_{l}$ can be written in the following general form as:

$\mathbf{f}_{l}=\left\{\begin{array}{lllllll}0 & \cdots & f_{l i} & \cdots & f_{l j} & \cdots & 0\end{array}\right\}^{T}$

where $f_{l}=f_{l i}\left(x_{l}, \dot{x}_{l}\right)=-f_{l j}\left(x_{l}, \dot{x}_{l}\right)$ depends only on the relative displacement $x_{l}$ and relative velocity $\dot{x}_{l}$ between two DOFs of the dispersed element.

\subsection{The equations of motion of the vehicle system}

The vehicle model, shown in Fig. 2, consists of a car-body, two bogies and four wheelsets joined by means of the primary and secondary suspensions. The wheelsets and bogies are considered as rigid bodies. Only vertical DOFs of vehicle are considered in this model. Each wheelset has vertical DOF, and each bogie has vertical and pitch DOFs. Modeling of the car-body and the primary suspension are described in Sects. 2.1 and 2.2. The secondary suspension is modeled as a linear spring plus a damper.

Combining the equations of motion of the wheelsets, the bogies, the car-body, the primary suspension and the secondary suspension and putting all the internal force vectors of the dispersed elements on the righthand-side, the equations of motion of the overall vehicle system can be written as

$\mathbf{M} \ddot{\mathbf{x}}+\mathbf{C \dot { x }}+\mathbf{K x}=-\sum_{l=1}^{N_{e}} \mathbf{f}_{l}+\sum_{k=1}^{4} \mathbf{p}_{w k}$ where $\ddot{\mathbf{x}}, \dot{\mathbf{x}}$ and $\mathbf{x}$ are the acceleration, velocity and displacement vectors, respectively, and $\mathbf{M}, \mathbf{C}$ and $\mathbf{K}$ represent the mass and viscous damping matrices and the linear part of the stiffness matrix of the vehicle system respectively. $\mathbf{p}_{w k}(k=1,2,3,4)$ denotes the wheeltrack excitation force acted on the $k$ th wheelset.

\subsection{The assumption of viscous damping}

It is noted that the left side of Eq. 9 represents a linear system. Its natural frequencies $\omega_{1}, \omega_{2}, \ldots, \omega_{h}$ and modal vectors $\boldsymbol{\Phi}_{1}, \boldsymbol{\Phi}_{2}, \ldots, \boldsymbol{\Phi}_{h}$ can be obtained by solving the generalized eigenvalue problem [20] as

$\left(\mathbf{M} \omega^{2}-\mathbf{K}\right) \boldsymbol{\Phi}=\mathbf{0}$

where $h$ is the number of modes required in the frequency range. The modal vectors can be normalized with respect to the mass matrix to yield the orthogonal conditions:

$\boldsymbol{\Phi}^{T} \mathbf{M} \boldsymbol{\Phi}=\mathbf{I} \quad \boldsymbol{\Phi}^{T} \mathbf{K} \boldsymbol{\Phi}=\boldsymbol{\Omega}^{2}$

$\boldsymbol{\Phi}=\left[\boldsymbol{\Phi}_{1}, \boldsymbol{\Phi}_{2}, \ldots, \boldsymbol{\Phi}_{h}\right]$

$\boldsymbol{\Omega}^{2}=\operatorname{diag}\left(\omega_{1}^{2}, \omega_{2}^{2}, \ldots, \omega_{h}^{2}\right)$

$\mathbf{I}=$ Identity matrix

Since viscous damping exists in the vehicle system, an assumption on the viscous damping matrix is needed to solve Eq. 9. The viscous damping matrix $\mathbf{C}$ in Eq. 9 is written as

$\boldsymbol{\Phi}^{T} \mathbf{C} \boldsymbol{\Phi}=\operatorname{diag}\left(2 \xi_{1} \omega_{1}, 2 \xi_{2} \omega_{2}, \ldots, 2 \xi_{h} \omega_{h}\right)$

where $\xi_{j}(j=1,2, \ldots, h)$ denotes the modal damping coefficients.

\section{Modified IHB method}

\subsection{Combining harmonic balance method with FRF}

The IHB method is a combination of the harmonic balance method (Ritz and Galerkin averaging method) and the incremental method (Newton-Raphson procedure) [5]. The first step in the IHB method is the harmonic balance. If the excitation force $\mathbf{p}_{w k}$ is assumed to be periodic, it can be expanded into Fourier series as:

$p_{w k}\{\tau\}=\sum_{n=0}^{\mathrm{NH}} P_{k n} \mathrm{e}^{\mathrm{i} n \tau} \quad k=1,2, \ldots, 4$ 
where $\tau=\omega t$ and $\mathrm{i}$ is the imaginary unit. Likewise, the solutions of Eq. 9 and the internal force vectors of the dispersed element $\mathbf{f}_{l}$ can also be expanded as:

$$
\begin{aligned}
x_{m}(\tau) & =\sum_{n=0}^{\mathrm{NH}} X_{m n} \mathrm{e}^{\mathrm{i} n \tau} \quad m=1,2, \ldots, N_{m} \\
f_{l}(\tau) & =\sum_{n=0}^{\mathrm{NH}} F_{l n} \mathrm{e}^{\mathrm{i} n \tau} \quad l=1,2, \ldots, N_{e}
\end{aligned}
$$

where $N_{m}$ denotes the total number of DOFs in the overall vehicle system and $\mathrm{NH}$ is the number of harmonic terms whose appropriate value depends on the desired accuracy. Substitute Eqs. 15 to 17 into Eq. 9 and the following equations can be obtained $[16,17]$ $\left(m=1,2, \ldots, N_{m}\right):$

$$
\begin{aligned}
X_{m n}= & -\sum_{l=1}^{N_{e}}\left(H_{m l_{i}}(n \omega)-H_{m l_{j}}(n \omega)\right) F_{l n} \\
& +\sum_{k=1}^{4}\left(H_{m k}(n \omega)\right) P_{k n}
\end{aligned}
$$

where $H_{m k}(n \omega)$ is the frequency response function (FRF) between the $m$ th DOF and the $k$ th DOF corresponding to the linear part of Eq. 9. The explicit formulas for $H_{m k}(n \omega)$ are given by Eq. 19, and they can be evaluated using the solutions of the eigenvalue problem obtained in Sect. 2.4.

$H_{m k}(n \omega)=\sum_{j=1}^{h} \frac{\Phi_{m j} \Phi_{\mathrm{kj}}}{-n^{2} \omega^{2}+2 \mathrm{i} n \xi_{j} \omega \omega_{j}+\omega_{j}^{2}}$

Equation 19 can be partitioned with unknowns related to the dispersed elements in one set of equation as

$\mathbf{X}_{1}=H_{11}(\omega) F\left(\mathbf{X}_{1}\right)+H(\omega) P$

and the other unknowns in the second set of equations as

$\mathbf{X}_{2}=H_{21}(\omega) F\left(\mathbf{X}_{1}\right)+H(\omega) P$.

The first partition related to the dispersed elements are independent (uncoupled) after combining the IHB method with the FRF. Therefore, only the unknowns related to the dispersed elements are needed to be solved with a much reduced computational cost. Select the DOFs connected to the dispersed elements and reform Eq. 18 as $\left(m=1,2, \ldots, N_{t}\right)$ :

$$
\begin{aligned}
X_{m n}= & -\sum_{l}^{N_{e}}\left(H_{m l 1}(n \omega)-H_{m l 2}(n \omega)\right) F_{l n} \\
& +\sum_{k}^{N_{p}}\left(H_{m k}(n \omega)\right) P_{k n}
\end{aligned}
$$

where $N_{t} \ll N_{m}$ represents the total number of DOFs related to the dispersed elements. Let

$$
\begin{aligned}
& \mathbf{c}_{m}=\left\{\begin{array}{llll}
X_{m 1} & X_{m 2} & \cdots & X_{m \mathrm{NH}}
\end{array}\right\}^{T} \\
& \mathbf{c}=\left\{\begin{array}{lllll}
\mathbf{c}_{1}^{T} & \mathbf{c}_{2}^{T} & \cdots & \mathbf{c}_{N_{t}}^{T}
\end{array}\right\}^{T} \\
& \mathbf{r}_{l}=\left\{\begin{array}{llll}
F_{l 1} & F_{l 2} & \cdots & F_{l \mathrm{NH}}
\end{array}\right\}^{T} \\
& \mathbf{r}=\left\{\begin{array}{llll}
\mathbf{r}_{1}^{T} & \mathbf{r}_{2}^{T} & \cdots & \mathbf{r}_{N_{e}}^{T}
\end{array}\right\}^{T} \\
& \mathbf{p}_{k}=\left\{\begin{array}{llll}
P_{l 1} & P_{l 2} & \cdots & P_{l \mathrm{NH}}
\end{array}\right\}^{T} \\
& \mathbf{p}=\left\{\begin{array}{lllll}
\mathbf{p}_{1}^{T} & \mathbf{p}_{2}^{T} & \cdots & \mathbf{p}_{N_{p}}^{T}
\end{array}\right\}^{T}
\end{aligned}
$$

where $c_{m}, r_{l}, p_{k}$ are the vectors of Fourier coefficients of displacement corresponding to the $m$ th DOFs, the $l$ th dispersed element and the $k$ th external force, respectively. Equation 20 can be expressed in matrix form as

$$
\begin{aligned}
& \mathbf{c}=-\mathbf{Y r}+\mathbf{Z} \mathbf{p} \\
& \mathbf{Y}=\left[\begin{array}{cccc}
Y_{11} & Y_{12} & \cdots & Y_{1 N_{e}} \\
Y_{21} & \cdots & \cdots & \cdots \\
\vdots & \vdots & \ddots & \vdots \\
Y_{N_{t} 1} & \cdots & \cdots & Y_{N_{t} N_{e}}
\end{array}\right] \\
& \mathbf{Z}=\left[\begin{array}{cccc}
Z_{11} & Z_{12} & \cdots & Z_{1 N_{p}} \\
Z_{21} & \cdots & \cdots & \cdots \\
\vdots & \vdots & \ddots & \vdots \\
Z_{N_{t} 1} & \cdots & \cdots & Z_{N_{t} N_{p}}
\end{array}\right] \\
& \mathbf{Y}_{m l}=\left[\begin{array}{lllll}
E_{m l}(0) & & & & \\
& E_{m l}(\omega) & & & \\
& & E_{m l}(2 \omega) & & \\
& & & \ddots & \\
& & & & E_{m l}(\mathrm{NH} \omega)
\end{array}\right] \\
& \mathbf{Z}_{m k}=\left[\begin{array}{lllll}
H_{m k}(0) & & & & \\
& H_{m k}(\omega) & & & \\
& & H_{m k}(2 \omega) & & \\
& & & \ddots & \\
& & & & H_{m k}(\mathrm{NH} \omega)
\end{array}\right]
\end{aligned}
$$

where $E_{m l}(n \omega)=H_{m l_{i}}(n \omega)-H_{m l_{j}}(n \omega)$. 
3.2 The incremental solution procedure

The second step to apply the IHB method is the well-known Newton-Raphson procedure [5]. It will be briefly explained here. Define the residual vector as

$\varepsilon=-\mathbf{c}-\mathbf{Y r}+\mathbf{Z} \mathbf{p}$

The residual vector will be null when in equilibrium, and Eq. 25 may be written as:

$\varepsilon=\mathbf{0}$

The new solutions $\mathbf{c}, \omega$ are obtained by adding the increments $\Delta \mathbf{c}, \Delta \omega$ to the current solutions $\mathbf{c}_{0}, \omega_{0}$ to have

$\mathbf{c}=\mathbf{c}_{0}+\Delta \mathbf{c} \omega=\omega_{0}+\Delta \omega$

The residual vector can be written as $\varepsilon_{0}=-\mathbf{c}_{0}-\mathbf{Y}\left(\omega_{0}\right) \mathbf{r}\left(\mathbf{c}_{0}, \omega_{0}\right)+\mathbf{Z}\left(\omega_{0}\right) \mathbf{p}$.

Expanding $\mathbf{Y r}$ and $\mathbf{Z p}$ in incremental form and neglecting the higher-order terms, the following formula can be obtained

$$
\begin{aligned}
\mathbf{Y r}= & \mathbf{Y}\left(\omega_{0}\right)\left[\mathbf{r}\left(\omega_{0}, \mathbf{c}_{0}\right)+\left.\frac{\partial \mathbf{r}}{\partial \omega}\right|_{\omega_{0}} \Delta \omega+\left.\frac{\partial \mathbf{r}}{\partial \mathbf{c}}\right|_{c_{0}} \Delta \mathbf{c}\right] \\
& +\left.\mathbf{r}\left(\omega_{0}, \mathbf{c}_{0}\right) \frac{\partial \mathbf{Y}}{\partial \omega}\right|_{\omega_{0}} \Delta \omega \\
\mathbf{Z p}= & \mathbf{Z}\left(\omega_{0}\right) \mathbf{p}+\left.\frac{\partial \mathbf{Z}}{\partial \omega}\right|_{\omega_{0}} \mathbf{p} \Delta \omega
\end{aligned}
$$

Substitute Eqs. 27 to 29 into Eq. 25, the incremental form of the residual vector can be written as:

$$
\begin{aligned}
\varepsilon= & \varepsilon_{0}-\left[\left.\mathbf{Y}\left(\omega_{0}\right) \frac{\partial \mathbf{r}}{\partial \mathbf{c}}\right|_{c_{0}}+\mathbf{I}\right] \Delta \mathbf{c} \\
& +\left[-\left.\mathbf{Y}\left(\omega_{0}\right) \frac{\partial \mathbf{r}}{\partial \omega}\right|_{\omega_{0}}-\left.\mathbf{r}\left(\omega_{0}, \mathbf{c}_{0}\right) \frac{\partial \mathbf{Y}}{\partial \omega_{\omega_{0}}}\right|_{\omega_{0}}\right. \\
& \left.+\left.\frac{\partial \mathbf{Z}}{\partial \omega}\right|_{\omega_{0}} \mathbf{p}\right] \Delta \omega
\end{aligned}
$$

where $\mathbf{r}_{l}, \frac{\partial \mathbf{r}}{\partial \mathbf{c}}$ and $\frac{\partial \mathbf{r}}{\partial \omega}$ can be written into explicit forms for the cubic nonlinear hardening springs and the dispersed dampers $[5,16,17]$. Note that $\varepsilon_{0}$ have vanished for a correct solution. Then the solutions of Eq. 24 can be obtained from the following iterative steps:

$$
\begin{aligned}
& -\left[\left.\mathbf{Y}\left(\omega_{i}\right) \frac{\partial \mathbf{r}}{\partial \mathbf{c}}\right|_{c_{i}}+\mathbf{I}\right] \Delta \mathbf{c}_{i+1} \\
& =\varepsilon_{i}+\left[\left.\mathbf{Y}\left(\omega_{i}\right) \frac{\partial \mathbf{r}}{\partial \omega}\right|_{\omega_{i}}\right. \\
& \left.\quad+\left.\mathbf{r}\left(\omega_{i}, \mathbf{c}_{i}\right) \frac{\partial \mathbf{Y}}{\partial \omega}\right|_{\omega_{i}}+\left.\frac{\partial \mathbf{Z}}{\partial \omega}\right|_{\omega_{i}} \mathbf{p}\right] \Delta \omega_{i+1} \\
& \mathbf{c}_{i+1}=\mathbf{c}_{i}+\Delta \mathbf{c}_{i+1} \\
& \omega_{i+1}=\omega_{i}+\Delta \omega_{i+1}
\end{aligned}
$$

One should note that the number of unknowns in Eq. 31 is larger than the number of equations for the presence of $\omega$. So either one of $\mathbf{c}$ and $\omega$ should be fixed to perform the above iterations. A converged solution is obtained when $\varepsilon_{0}$ reaches a prescribed small error. Then using the current converged solution as an initial condition and prescribing either one of $\Delta \mathbf{c}$ and $\Delta \omega$ to a small value, the iterative process will begin for the next solution. The steady-state solution of the nonlinear system can then be obtained for different frequency points.

\section{Simulations}

Parameters in the following vehicle modeling are based on the CRH3 trains in China. According to the mode selection criteria introduced by Carlbom [2,3], 53 vibration modes of the vehicle system are selected. Since the natural frequency of the primary suspension springs is close to the third bending modal frequency in this vehicle system, the interaction between those two modes and the responses of the vehicle system in the vicinity of natural frequency of the third bending mode are of most concern in this study. All the frequencies are normalized by the third bending modal frequency. Table 1 lists the dimensionless natural frequencies of the first five bending modes of the vehicle system.

The periodic wheel-track excitations of the vehicle system are applied vertically on the wheelsets as

$\mathbf{P}_{w k}=P \cos (\omega t) \quad k=1,2,3,4$

Table 1 The natural frequencies of first five bending mode of the carbody

\begin{tabular}{llllll}
\hline set $i$ & 1 & 2 & 3 & 4 & 5 \\
\hline$\omega_{c i} / \omega_{c 3}$ & 0.33 & 0.66 & 1 & 1.41 & 1.98 \\
\hline
\end{tabular}




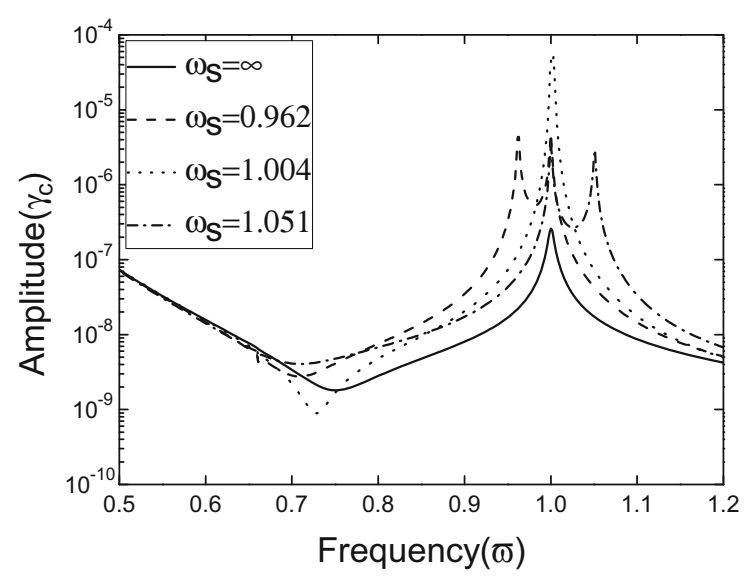

Fig. 3 Vertical amplitude response of the car-body centroid $(\beta=$ $\left.0, \xi_{0}=0.001\right)$

and the following dimensionless quantities are defined as

$$
\begin{gathered}
\alpha=\frac{P}{W}, \omega_{s}=\frac{\bar{\omega}}{\omega_{c 3}}, \varpi=\frac{\omega}{\omega_{c 3}}, \\
\gamma_{c}=z_{c} / x_{0}, \gamma_{b}=z_{b} / x_{0} .
\end{gathered}
$$

where the axle load is denoted by $W$. The excitation frequency and the fundamental natural frequency of the primary suspension spring are denoted by $\omega$ and $\bar{\omega}$ respectively. Symbol $\gamma_{c}=z_{c} / x_{0}$ and $\gamma_{b}=$ $z_{b} / x_{0}$ represent the amplitude of responses of the centroid of the car-body and the bogie in the vertical direction. All of modal damping ratios are assumed identical and equal to $\xi_{0}$. According to experimental and simulated results $[9,18]$, the amplitude of the contact forces oscillate between $1 / 60$ and $1 / 10$ of the axle load. So the dimensionless excitation amplitude $\alpha$ remains unchanged and equals to $1 / 30$ in this study.

\subsection{The influence of dynamic response of the primary suspension}

The primary suspension springs are assumed to be linear in this section with $\beta=0$. With changing $\bar{\omega}$, the vertical responses of the car-body centroid and the bogie are shown in Figs. 3 and 4, respectively. $\omega_{s}=\infty$ in these figures corresponds to the case where the general static model is adopted for the primary suspension springs with the dynamic effects ignored. Figure 3 shows that the dynamic response of the primary suspension spring has no effect when the excitation fre-

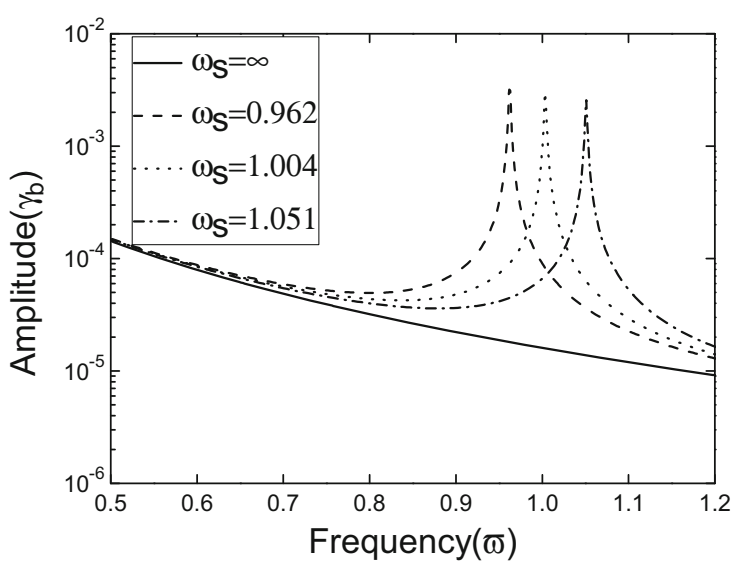

Fig. 4 Vertical amplitude response of the bogie $\left(\beta=0, \xi_{0}=\right.$ $0.001)$

quency is away from the fundamental natural frequency of the primary suspension springs. For the static model, the response curve has only one peak corresponding to the frequency $\omega_{c 3}$. Since the car-body rests on the secondary suspension located exactly at the node of the second bending mode, there is no peak in the vicinity of the second bending mode of the vehicle system. The response curve for the dynamic model has two peaks with one corresponding to the frequency $\omega_{c 3}$ and the other one corresponds to $\bar{\omega}$. The peak response amplitude of the car-body centroid depends on $\omega_{s}$. When $\bar{\omega}$ is very close to $\omega_{c 3}$, the peak amplitude increases and reaches the maximum value at $\omega_{s}=1$. When $\omega_{s}$ is in the vicinity of 1.0 , say $\omega_{s}=1.004$, the peak amplitude is 15 times larger than that from the static model. It may be concluded that if the natural frequency of the primary suspension springs gets closer to a higher modal frequency of the vehicle system, the dynamic response of the primary suspension springs has significant effect on the response of the carbody.

Figure 4 shows the amplitude of responses of the bogies. For the case with the static model, the response curve is descending with increasing $\varpi$ indicating that the high frequency excitation cannot be transmitted from the wheelsets to the bogies. However, the amplitude of responses from the dynamic model is much larger in the vicinity of $\bar{\omega}$. It may be concluded that the vibration of the bogie is amplified when the excitation frequency is in the vicinity of the natural frequency of the suspension spring and the vibration is transmitted to the bogies. 


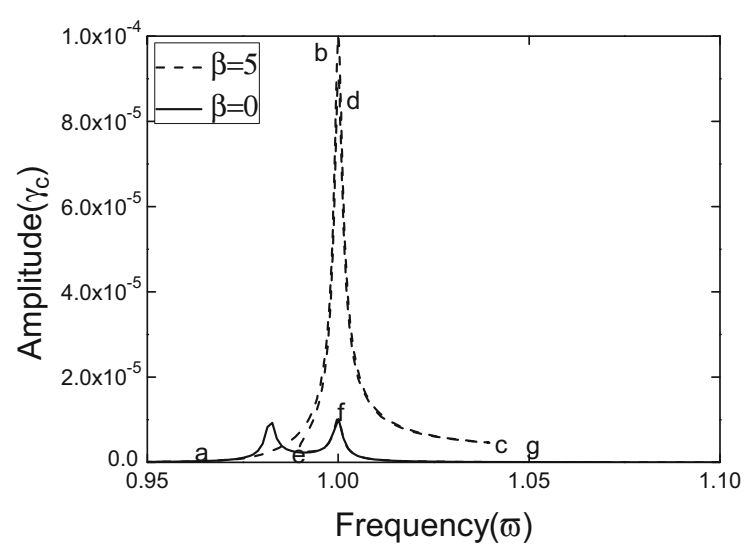

Fig. 5 Vertical amplitude response of the car-body centroid $\left(\omega_{s}=0.98, \xi_{0}=0.001\right)$

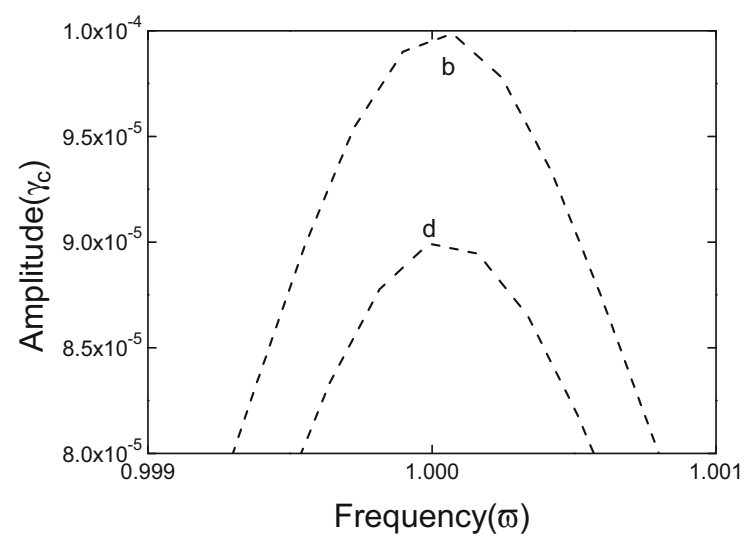

Fig. 6 Vertical amplitude response of the car-body centroid (partial enlarged $\omega_{s}=0.98, \xi_{0}=0.001$ )

\subsection{The influence of nonlinearity of the primary suspension springs}

To study the case when the natural frequency of the third order bending natural frequency is close to the natural frequency of the primary suspension springs, the parameter $\omega_{s}$ is set equal to 0.98 . The response of the vehicle system is stimulated with linear and nonlinear primary suspension springs ( $\beta=0$ and $\beta=5$ ). Figure 5 shows the comparison of the vertical response of the car-body centroid in the two cases. Figure 6 is partial enlarged in the vicinity of the peak value. The linear case $(\beta=0)$ with solid line has only one branch going along the route $a \rightarrow f \rightarrow g$. The nonlinear case $(\beta=5)$ with dotted line has two branches. One goes along the route $a \rightarrow b \rightarrow c$ and the other goes along the route $c \rightarrow d \rightarrow e \rightarrow f \rightarrow g$. It can be seen

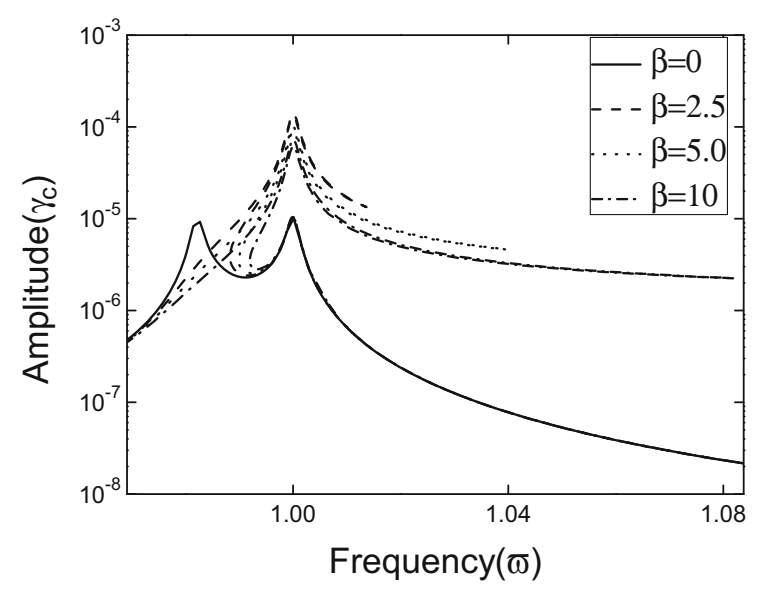

Fig. 7 Vertical amplitude response of the car-body centroid $\left(\omega_{s}=0.98, \xi_{0}=0.001\right)$

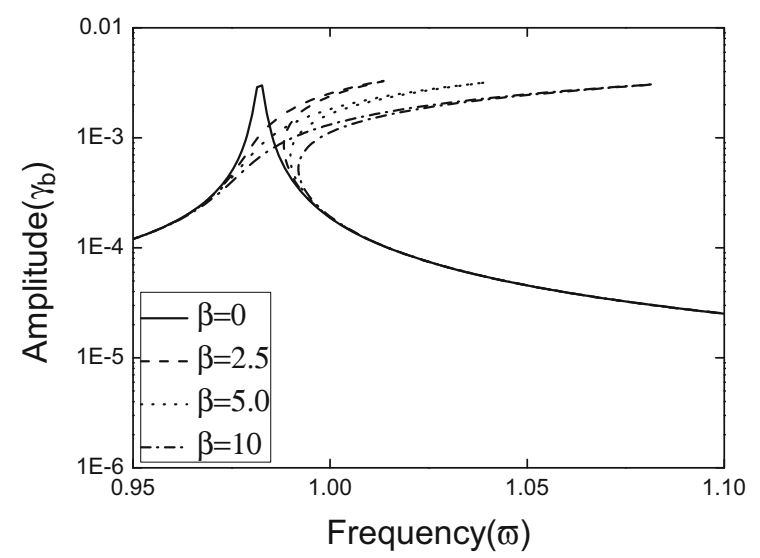

Fig. 8 Vertical amplitude response of the bogies $\left(\omega_{s}=0.98\right.$, $\left.\xi_{0}=0.001\right)$

that the response are identical when the excitation frequency is away from the frequency $\bar{\omega}$. But the results are totally different in the vicinity of the frequency $\bar{\omega}$. This is because the excitation frequency, the natural frequency of the flexural mode of the vehicle system and the inherent frequency of the suspension system are in close proximity. Due to nonlinearity of the suspension springs, the vibration energy transmits between the third natural bending mode of the vehicle system and the first natural mode of the primary suspension system. In this case, the vehicle system would generate the internal resonance and the response in the vicinity of the frequency $\bar{\omega}$ is called internal resonance response.

Figures 7 and 8 compare the vertical responses of the car-body centroid and the bogies with different parameter $\beta$. The curve would bend to right more obvi- 


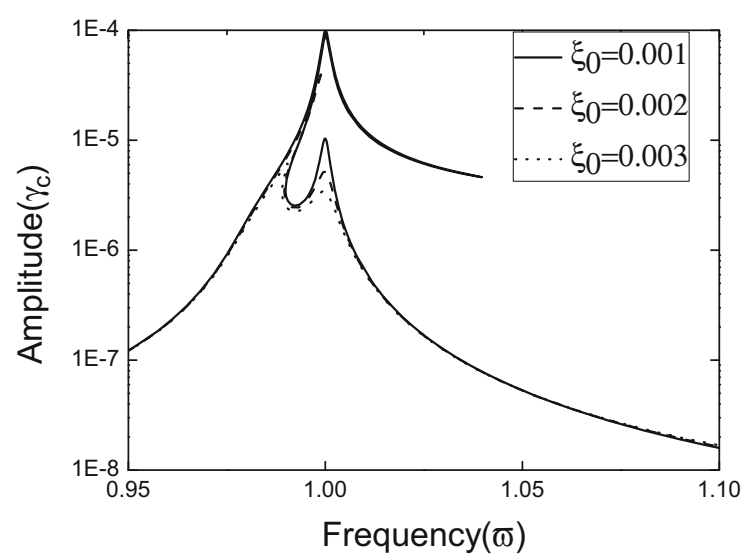

Fig. 9 Vertical amplitude response of the car-body centroid $\left(\omega_{s}=0.98, \beta=5\right)$

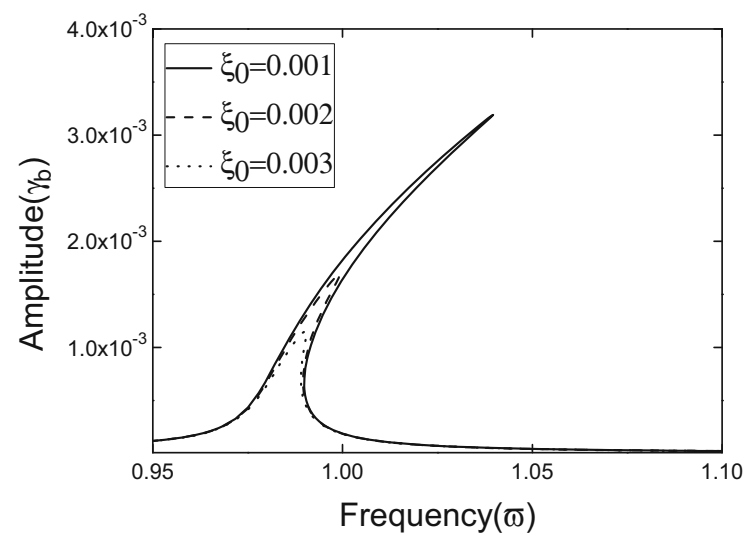

Fig. 10 Vertical amplitude response of the bogies $\left(\omega_{s}=0.98\right.$, $\beta=5$ )

ously with bigger $\beta$ and the internal resonance response occurs over a wider frequency range. It may be concluded that the nonlinearity of the primary suspension spring is a key factor for the occurrence of internal resonance.

\subsection{The influence of the modal damp ratio}

To study the effect of the modal damping ratio on the internal resonance vibration, the parameter $\xi_{0}$ is varied from 0.001 to 0.003 . The amplitude responses of the car-body centroid and the bogies are shown in Figs. 9 and 10 respectively.

The results show that the internal resonance will become weaker with an increase of $\xi_{0}$. The internal resonance almost disappears when $\xi_{0}=0.003$. There- fore, the damping ratio of vehicle system is also a key parameter for the occurrence of internal resonance.

\section{Conclusions}

The equations of motion of a high-speed vehicle system including the dynamic effects of the primary suspension and the flexibility of the car-body have been derived in this paper. The steady-state responses of the high-speed vehicle system under periodic excitations are solved with an approximate harmonic approach incorporating the IHB method with FRF in the frequency domain. Since the algorithm only solves the equations of the unknowns related to the dispersed internal elements, it offers an effective means to analyze the dynamic behavior of a vehicle system with local nonlinearity.

If the wheel-rail excitation frequencies come close to the fundamental natural frequency of the primary suspension with increasing running speed, it is found that the dynamic response of the primary suspension spring has a great influence on the responses of the high-speed vehicle whether the primary suspension spring is linear or nonlinear. When the natural frequency of the primary suspension springs gets closer to one of the higher modal frequencies of the vehicle system, the internal resonance which involves the fundamental mode of the primary suspension spring and the higher modes of the vehicle system would have occurred. And then the responses of the high-speed vehicle system would have been amplified. This is especially true for the nonlinear case. It may be concluded that the nonlinearity of the primary suspension spring and the damping ratios of the vehicle system both play very important roles for the occurrence of internal resonance. A stronger nonlinear hardening of the primary suspension springs or a smaller modal damping ratio would lead to internal resonance more easily over a wider range of frequency.

Acknowledgements This work was supported by a grant from the Major State Basic Research Development Program of China (973 Program, No. 2011CB711100) and a grant from the National Key Research and Development Plan of China (No. 2016YFC0701302).

\section{References}

1. Bruni, S., Vinolas, J., Berg, M., Polach, O., Stichel, S.: Modelling of suspension components in a rail vehicle dynamics context. Veh. Syst. Dyn. 49(7), 1021-1072 (2011) 
2. Carlbom, P.F.: Carbody and passengers in rail vehicle dynamics. Ph.D. Thesis, KTH (2000)

3. Carlbom, P.F.: Combining MBS with FEM for rail vehicle dynamics analysis. Multibody Syst. Dyn. 6(3), 291-300 (2001)

4. Chen, S., Cheung, Y., Xing, H.: Nonlinear vibration of plane structures by finite element and incremental harmonic balance method. Nonlinear Dyn. 26(1), 87-104 (2001)

5. Cheung, Y., Chen, S., Lau, S.: Application of the incremental harmonic balance method to cubic non-linearity systems. J Sound Vib. 140(2), 273-286 (1990)

6. Escalona, J.L., Sugiyama, H., Shabana, A.A.: Modelling of structural flexiblity in multibody railroad vehicle systems. Veh. Syst. Dyn. 51(7), 1027-1058 (2013)

7. Xue, H., Li, Y.C., Liu, W.X.: The test method research of the coil spring of locomotive and rolling stock. J. Lanzhou Jiaotong Univ. 4, 030 (2013)

8. Lau, S., Cheung, Y.: Amplitude incremental variational principle for nonlinear vibration of elastic systems. J. Appl. Mech. 48(4), 959-964 (1981)

9. Lee, H., Sandu, C., Holton, C.: Dynamic model for the wheel-rail contact friction. Veh. Syst. Dyn. 50(2), 299-321 (2012)

10. Lee, J., Thompson, D.: Dynamic stiffness formulation, free vibration and wave motion of helical springs. J. Sound Vib. 239(2), 297-320 (2001)

11. Liu, L., Zhang, W.H.: Frequency variety analysis and equivalent algorithm of metal spring stiffness. J. Traffic Transp. Eng. 7(5), 24-27 (2007). in Chinese

12. Liu, L., Zhang, W.H.: Study on dynamic characteristics of metal helical spring. Sci. Pap. Online 2(9), 674-684 (2007). in Chinese

13. Lu, C.J., Lin, Y.M.: A modified incremental harmonic balance method for rotary periodic motions. Nonlinear Dyn. 66(4), 781-788 (2011)
14. Pierre, C., Dowell, E.: A study of dynamic instability of plates by an extended incremental harmonic balance method. J. Appl. Mech. 52(3), 693-697 (1985)

15. Popp, K., Kruse, H., Kaiser, I.: Vehicle-track dynamics in the mid-frequency range. Veh. Syst. Dyn. 31(5-6), 423-464 (1999)

16. Pun, D., Lau, S., Liu, Y.: Internal resonance of an 1-shaped beam with a limit stop: Part 1, free vibration. J. Sound Vib. 193(5), 1023-1035 (1996)

17. Pun, D., Lau, S., Liu, Y.: Internal resonance of an 1-shaped beam with a limit stop: Part 2. forced vibration. J. Sound Vib. 193(5), 1037-1047 (1996)

18. Shabana, A.A., Zaazaa, K.E., Escalona, J.L., Sany, J.R.: Development of elastic force model for wheel/rail contact problems. J. Sound Vib. 269(1), 295-325 (2004)

19. Sze, K., Chen, S., Huang, J.: The incremental harmonic balance method for nonlinear vibration of axially moving beams. J. Sound Vib. 281(3), 611-626 (2005)

20. Thomson, W.: Theory of Vibration with Applications. CRC Press, Boca Raton (1996)

21. Liu, Y.B., Zhang, Y.Y.: Coherence analysis on the vehicle vibration response and track irregularities. In: Proceedings of the twenty second national conference on structural engineering, Part I. (2013) (in Chinese)

22. Zhang, S.G., Kang, X., Liu, X.: Characteristic analysis of the power spectral density (PSD) of track irregularity on beijingtianjin inter-city railway. China Railway. Science 29(5), 2530 (2008)

23. Zhang, Y.H., Liu, H.H., Wang, D.C.: Manual of Spring. China Machine Press, Beijing (1997). in Chinese

24. Zhou, J., Goodall, R., Ren, L., Zhang, H.: Influences of car body vertical flexibility on ride quality of passenger railway vehicles. Proc. Inst. Mech. Eng. Part F J. Rail Rapid Transit 223(5), 461-471 (2009) 